\title{
Proceedings of the 8th International Conference on Security of Information and Networks
}

September 8-10, 2015 Sochi/Russia
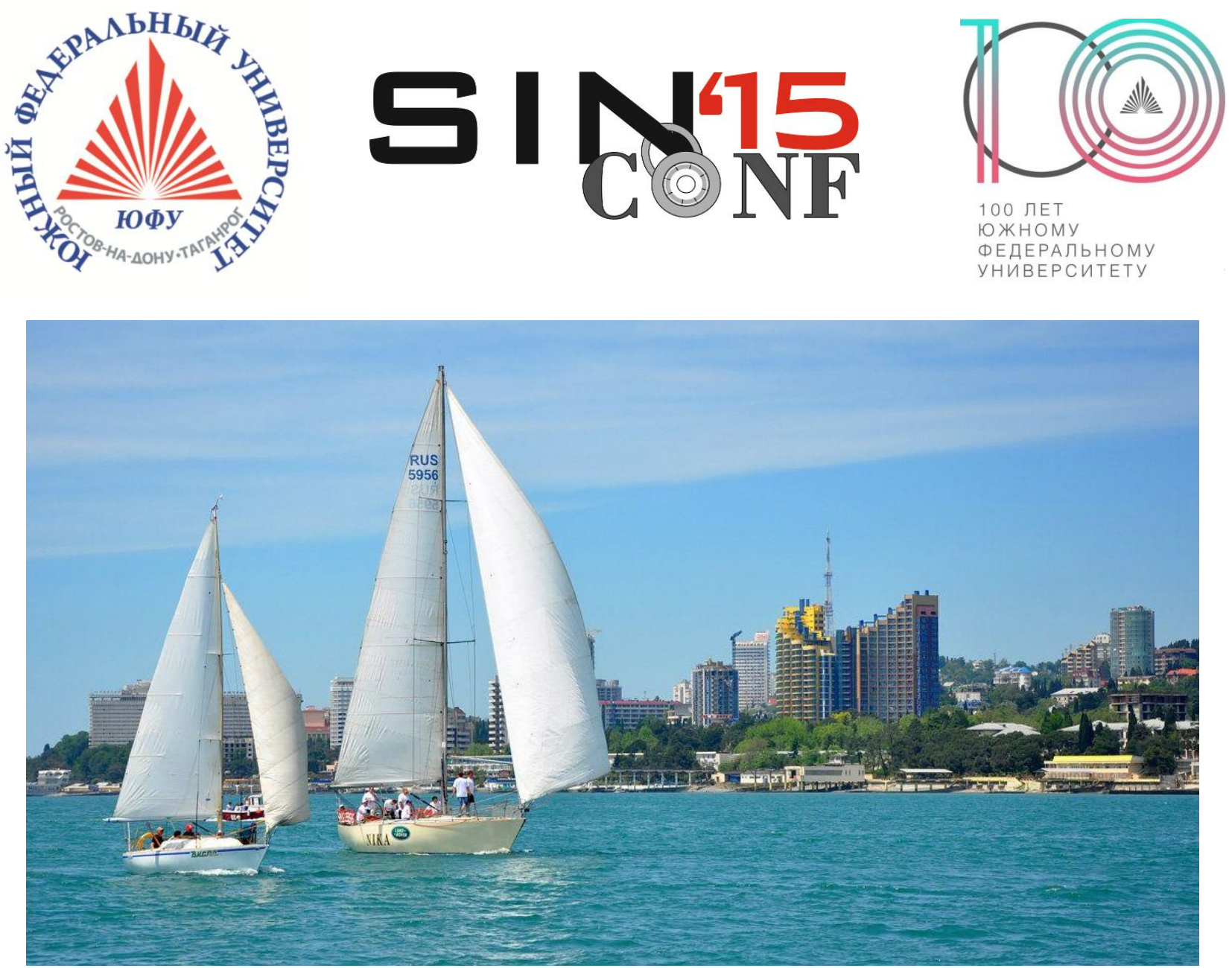

Organized by:

- Southern Federal University, Taganrog, Rostov-on-Don, Russia

- The University of Glasgow, Glasgow, UK

- Aksaray University, Aksaray, Turkey

- Malaviya National Institute of Technology, Jaipur, India

- Macquarie University, Sydney, Australia

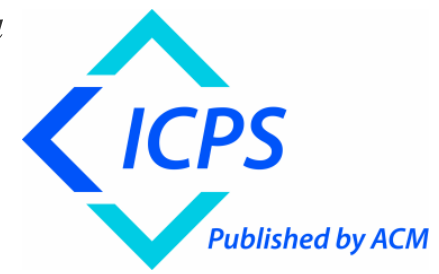

Edited by Oleg Makarevich, Ludmila Babenko, Maxim Anikeev, Ronald Poet, Atilla Elçi, Manoj Singh Gaur \& Mehmet Orgun 


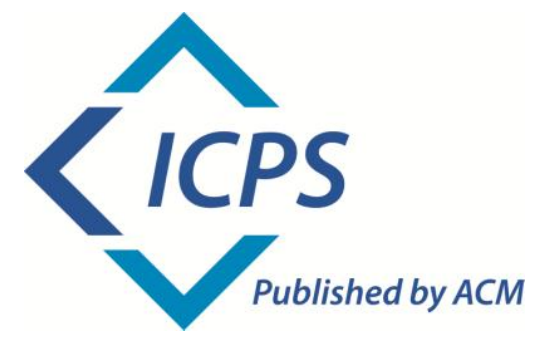

The Association for Computing Machinery

2 Penn Plaza, Suite 701

New York New York 10121-0701

ACM COPYRIGHT NOTICE. Copyright $\odot 2015$ by the Association for Computing Machinery, Inc.

Permission to make digital or hard copies of part or all of this work for

personal or classroom use is granted without fee provided that copies are not made or

distributed for profit or commercial advantage and that copies bear this notice and the

full citation on the first page. Copyrights for components of this work owned by others

than ACM must be honored. Abstracting with credit is permitted. To copy otherwise, to

republish, to post on servers, or to redistribute to lists, requires prior specific permission

and/or a fee. Request permissions from Publications Dept., ACM, Inc., fax +1 (212) 869-0481, or permissionseacm. org.

For other copying of articles that carry a code at the bottom of the first or last page, copying is permitted provided that the per-copy fee indicated in the code is paid through the Copyright Clearance Center, 222 Rosewood Drive, Danvers, MA 01923, $+1-978-750-8400,+1-978-750-4470$ (fax).

ACM ISBN: 978-1-4503-3453-2 


\section{Foreword}

Dear colleagues and friends, we invite you to the $8^{\text {th }}$ International Conference on Security of Information and Networks (SIN 2015), which is held in Sochi, one of the most beautiful cities in Russia. In 2014, Sochi held remarkable Winter Olympic Games. You will be inspired by warm Black Sea, high Caucasus, and interesting discussions of information security problems. The conference of SIN series is held in Russia for the second time. It is hosted by Southern Federal University (SFedU), in particularly by its following subdivisions: the Institute of Computing Technologies and Information Security, the Department of IT-Security, and South-Russian Regional Educational and Research Center for IT-Security Problems. The honorary chair of the conference is Dr. Marina Borovskaya, the rector of SFedU.

The $8^{\text {th }}$ International Conference on Security of Information and Networks is an international forum for presentation of theoretical and applied results in the area of information and network security. SIN 2015 continues the tradition of fruitful meetings of SIN series in Famagusta, Taganrog, Sydney, Jaipur, Aksaray, and Glasgow. Future conferences are scheduled to take place in the USA (2016) and Australia (2017). The program of SIN 2015 includes invited and selected peerreviewed talks, as well as special sessions on theory and practice of information security. The papers will be published in the digital library of Association of Computing Machinery (ACM) and indexed by Scopus. Just like previous events of SIN series, SIN 2015 is carried out in technical cooperation with Special Interest Group on Security Audit and Control of ACM. The Program Committee received 92 paper proposal, of which 34 were accepted as full papers and 29 were accepted as short papers, position statements and fast abstracts. Geographical distribution of the participants is quite diverse. There are 37 representatives of 16 Russian universities, including Southern Federal University, National Research Nuclear University MEPhI, Bauman Moscow State Technological University, Kazan National Research Technological University, Peter the Great St. Petersburg Polytechnic University, Novosibirsk State University, Omsk State University of Technology, Siberian State Aerospace University, Samara State University of Technology, etc. Nearly thirty participants of the conference represent universities and research institutions of Belgium, China, Colombia, Germany, India, Iran, Italy, Luxembourg, Pakistan, Tunisia, Turkey, the United Kingdom, and the USA.

The following keynote talks are invited to the conference: "Lightweight Cryptography: Modern Development Paradigms" by Alexey Zhukov (Bauman Moscow State Technological University); "The Use of Mobile Devices in Authentication" by Ron Poet (The University of Glasgow); "Record Linkage Applications in Health Services Research: Opportunities and Challenges" by Ramakrishna Thurimella, Rinku Dewri and William Mitchell (The University of Denver); and "Modern Trends in the Regulatory Framework of the Information Security Compliance Assessment in Russia Based on Common Criteria" by Alexey Markov and Alexander Barabanov (Echelon, Moscow).

The Organizing Committee would like to thank Russian Foundation for Basic Research, NeoBit LLC and NPO Echelon for their financial support. We are very grateful to all the authors, reviewers, organizers and volunteers for their contributions to the quality of the conference.

Oleg Makarevich

Ludmila Babenko

Maxim Anikeev
Ron Poet

Atilla Elçi
Manoj Singh Gaur

Mehmet Orgun 


\section{Table of Contents}

\section{Keynote Sessions}

- The use of mobile devices in authentication

Ron Poet (University of Glasgow)

- Record Linkage Applications in Health Services Research: Opportunities and

Challenges.

Ramakrishna Thurimella (University of Denver)

- Lightweight Cryptography: Modern Development Paradigms 7

Alexey Zhukov (Bauman Moscow State Technical University)

\section{Session A.1: "Security in information systems"}

- Information Security Risk Management in Computer Networks Based on Fuzzy Logic and Cost/Benefit Ratio Estimation.

Igor Anikin, Lilia Emaletdinova (KNRTU Kazan)

- Analysis of the Impact of Ethical Issues on the Management of the Access Rights.

Andre Rifaut, Christophe Feltus, Slim Turki, Djamel Khadraoui (Luxembourg Institute of Science and Technology)

- sandFOX: Secure Sandboxed and Isolated Environment for Firefox Browser

Anil Saini, Manoj Gaur, Vijay Laxmi (MNIT Jaipur), Priyadarsi Nanda (UTS Australia)

\section{Session A.2: "Security in information systems"}

- Modern Techniques of Function-Level Fault Tolerance in MFM-Systems 28 Alexander Tarasov (NPO Echelon)

- Modern Trends in the Regulatory Framework of the Information Security Compliance Assessment in Russia Based on Common Criteria

Alexey Markov (NPO Echelon), Alexander Barabanov (Bauman MSTU)

- Problems of Data Protection in Industrial Corporations Enterprise Architecture

Vladimir Glukhov, Igor Ilin, Alexey Anisiforov (Peter the Great St. Petersburg Polytechnic University)

- Secure e-Learning Portal for Teaching Business Continuity and Information Security Management.

Natalia Miloslavskaya, Alexander Tolstoy, Vladislav Petrov (National Research Nuclear University (MEPhI))

- Access control model D-TBAC subject to the requirements to tasks' performing

Sergey Lapin (Altai State University)

\section{Session A.3: "Security in information systems"}

- An Analytical Processing Approach to Supporting Cyber Security Compliance Assessment

Francesco Buccafurri, Lidia Fotia (University of Reggio Calabria), Angelo Furfaro, Alfredo Garro

(University of Calabria), Matteo Giacalone (Poste Italiane), Andrea Tundis (University of Calabria)

- Building data in motion DLP system from scratch using opensource software and confirming its effectiveness within 'Capture The Flag' competitions

Artem Garkusha (Southern Federal University) 


\section{Session B.1: "Cryptographic techniques and key management"}

- A Perfect Dynamic-Id and Biometric Based Remote User Authentication Scheme Under Multi-Server Environments Using Smart Cards.

Subhasish Banerjee, Manash Dutta, Chandan Bhunia (NIT Arunachal Pradesh)

- Absolute Key Variation Technique of Automatic Variable Key in Cryptography

Rajat Goswami, Subhasish Banerjee, Manash Dutta, Chandan Bhunia (NIT Arunachal Pradesh)

- Improved Trust Based Key Management for End-to-End Secure Communication in Cellular Networks

Naila Mukhtar (Center for Advanced Studies in Engineering Islamabad)

\section{Session B.2: "Cryptographic techniques and key management"}

- Secure Voice Communication System with Hardware Encryption of Data on Hands-Free Headset

Alexander Boruchinkin (National Research Nuclear University (MEPhI))

- Cryptanalysis of Factoring-based Fully Homomorphic Encryption

Alina Trepacheva, Lyudmila Babenko (Southern Federal University)

- Execution of Data-Dependent Programs Over Encrypted Data

Philipp Burtyka, Oleg Makarevich (Southern Federal University)

- Improvement of Treeless Signature Schemes Implementation by Random Oracle

Buffering

Maxim Anikeev (Southern Federal University)

\section{Session C.1: "Security-aware software engineering"}

- Synthesis of Secure Software Development Controls.

Alexey Markov (NPO Echelon), Valentin Tsirlov (Bauman MSTU), Alexander Barabanov, Andrey

Fadin (Bauman MSTU), Igor Shakhalov (NPO Echelon)

- A Production Model System for Detecting Vulnerabilities in the Software Source Code .98 Alexey Markov (NPO Echelon), Valentin Tsirlov, Alexander Barabanov, Andrey Fadin (Bauman MSTU)

\section{Session C.Special: "Detecting and Preventing Fraud with Data Analytics"}

- Approbation of the methodology for Web monitoring of terrorism- and extremism-related content

Natalia Evstifeeva, Olga Evstifeeva (National Research Nuclear University (MEPhI))

- Application of Cluster Analysis for the Assessment of the Share of Fraud Victims among Bank Card Holders

Stanislav Alkhasov, Alexander Tselykh, Alexey Tselykh (Southern Federal University)

- Profile-Based Students Assignment to Core Financial Intelligence Unit Departments

Daria Chukova, Pakhomov Alexey (National Research Nuclear University (MEPhI))

- A Graph-Based Data Mining Approach to Preventing Financial Fraud: A Case Study Margarita Knyazeva, Alexander Tselykh, Alexey Tselykh, Elena Popkova (Southern Federal University)

- Web Service for Detecting Credit Card Fraud in Near Real-Time

Alexey Tselykh (Southern Federal University), Dmitry Petukhov (Immanuel Kant Baltic Federal University)

- Mitigating Conflicts of Interest by Authorization Policies

Nezar Nassr, Eric Steegmans (KU Leuven) 


\section{Session A.Special.1: "Cybersecurity of Large-Scale and Distributed systems"}

- Information Security in Computer Networks with Dynamic Topology .

Aleksandr Minin, Maxim Kalinin (Peter the Great St. Petersburg Polytechnic University)

- Steganographic Methods of Communications in Distributed Computing Networks.

Artem Konoplev, Alexey Busygin (Peter the Great St. Petersburg Polytechnic University)

- Large-scale Systems Security Evolution: Control Theory Approach.

Dmitry Zegzhda, Tatiana Stepanova (Peter the Great St. Petersburg Polytechnic University)

- Ontology-based Big Data Approach to Automated Penetration Testing of Large-scale Heterogeneous Systems

Tatiana Stepanova, Alexander Pechenkin, Daria Lavrova (Peter the Great St. Petersburg Polytechnic University)

- Cybersecurity of Ad-hoc Distributed Systems

Denis Ivanov (NeoBIT LLC), Dmitry Moskvin (Peter the Great St. Petersburg Polytechnic University)

\section{Session A.Special.2: "Cybersecurity of Large-Scale and Distributed systems"}

- Towards Proactive Detection of Advanced Persistent Threat (APT) Attacks using Honeypots

Zainab Saud, Dr M Hasan Islam (Center for Advanced Studies in Engineering Islamabad)

- A honeypot-driven cyber incident monitor: lessons learned and steps ahead

Emmanouil Vasilomanolakis, Shankar Karuppayah (TU Darmstadt), Panayotis Kikiras

(AGT International), Max Muehlhauser (TU Darmstadt)

- A Classification Framework for Distinct Cyber-Attacks based on Occurrence Patterns

Malik S. K. Awan (Cardiff University), Mohammed AlGhamdi, Sultan AlMotiri (Umm AlQura

University), Pete Burnap, Omer Rana (Cardiff University)

- DDoS/EDoS attack in Cloud: Affecting everyone out there!

Gaurav Somani, Manoj Gaur (MNIT Jaipur), Dheeraj Sanghi (IIT Kanpur)

- The General Model of Secure Computation System

Philipp Burtyka, Oleg Makarevich, Lyudmila Babenko, Alina Trepacheva (Southern Federal University)

- Two formal problems of network security and a theory of Boolean-valued flow networks .. 185 Evgeny Shcherba (Omsk State Technical University)

\section{Session B.3.1: "Network security and protocols"}

- Developing a Penetration Test Methodology in Ensuring Router Security and Testing it in a Virtual Laboratory

Mehmet Yalcinkaya, Ecir Kucuksille, Samet Ganal (Suleyman Demirel University)

- Protection from Binary and Multi-Symbol Packet Length Covert Channels

Anna Epishkina, Konstantin Kogos (National Research Nuclear University (MEPhI))

- Trust management system for mobile cluster-based wireless sensor network Evgeny Abramov, Elena Basan, Oleg Makarevich (Southern Federal University)

- A New Algorithm for Unkeyed Jam Resistance

Hamid Hanifi (University of Denver), Leemon Baird, Ramakrishna Thurimella (University of Denver)

- Simulation User Behavior on A Security Testbed Using User Behavior States Graph Aragats Amirkhanyan, Andrey Sapegin, Marian Gawron, Feng Cheng, Christoph Meinel (Hasso Plattner Institute) 


\section{Session B.3.2: "Network security and protocols"}

- Development of network security tools for enterprise software-defined networks 224

Alexander Shukhman, Petr Polezhaev, Yuri Ushakov, Leonid Legashev (Orenburg State University), Veniamin Tarasov, Nadezhda Bakhareva (Volga Region State University of Telecommunications and Informatics))

- Automatic Vulnerability Detection for Weakness Visualization and Advisory Creation .229 Marian Gawron, Aragats Amirkhanyan, Feng Cheng, Christoph Meinel (Hasso Plattner Institute)

- Few Notes Towards Making Honeyword System More Secure and Usable

Nilesh Chakraborty, Samrat Mondal (IIT Patna)

\section{Session C.2: "Computational intelligence techniques in security"}

- Dynamic response recognition by neural network to detect network host anomaly activity

Vladimir Eliseev (Infotecs JSC), Yury Shabalin (National Research University (MPEI), Alfa Bank JSC)

- Modeling of Next-Generation Firewalls as Queueing Services

Sergey Zapechnikov, Natalia Miloslavskaya, Alexander Tolstoy (National Research Nuclear University $(M E P h I))$

- SQL-IDS: Evaluation of SQLi Attack Detection and Classification Based on Machine Learning Techniques.

Naghmeh Moradpoor (Abertay university)

- Designing a Context-Aware Cyber Physical System for Detecting Security Threats in Motor Vehicles

Andrei Petrovski, Prapa Ratadilok (Robert Gordon University Aberdeen), Sergei Petrovski (Samara State Technical University)

- Machine Learning Approach for Filtering Spam Emails

Vinod P. (SCMS College Kerala), Princy George (SCMS College Kerala)

\section{Session B.4: "Malware analysis"}

- A Dynamic Marking Method for Implicit Information Flow in Dynamic Taint Analysis 275 Xuefei Wang, Hengtai Ma, Lisha Jing (Institute of Software Chinese Academy of Sciences)

- DRACO:DRoid Analyst COmbo An Android Malware Analysis Framework .283 Shweta Bhandari, Rishabh Gupta, Vijay Laxmi, Manoj Singh Gaur (MNIT Jaipur), Akka Zemmari (LaBRI - University of Bordeaux), Maxim Anikeev (Southern Federal University)

- A Robust Dynamic Analysis System Preventing SandBox Detection by Android Malware ...290 Jyoti Gajrani, Jitendra Sarswat, Meenakshi Tripathi, Vijay Laxmi, Manoj Gaur (MNIT Jaipur), Mauro Conti (University of Padua)

- Information Theoretic Method for Classification of Packed and Encoded Files Vinod P. (SCMS College Kerala), Jithu Raphel (SCMS College Kerala)

- Hartley's Test Ranked Opcodes for Android Malware Analysis 304 Vinod P., Meenu Mary John, Dhanya K.A. (SCMS School of Engineering \& Technology Kerala)

\section{Session C.3: "Security algorithms and analysis"}

- Case based analysis in information security incidents management system

Andrey Shalyapin, Vadim Zhukov (Siberian State Aerospace University)

- Comparison between Safety and Efficient Security of the ARP Protocol

Elvia Leon (Universidad Nacional de Colombia), Brayan S. Reyes Daza (UDFJC Bogota), Octavio

Salcedo Parra (UDFJC Bogota) 
- DynaDroid: Dynamic Binary Instrumentation based App Behavior monitoring Framework. 322 Bharat Buddhdev, Rati Bhan, Manoj Singh Gaur, Vijay Laxmi (MNIT Jaipur)

- Developing a System for Text-Messages Protection 326

Dmitry Mikhaylov, Andrey Starikovskiy, Grigoriy Lebedev, Dmitry Rubin, Alexey Egorov, Eugeniy Uleykin, Anastasia Tolstaya, Alexander Boruchinkin (National Research Nuclear University (MEPhI))

- Labelled Mobile Ambients Model for Information Flow Security in Distributed Systems ..... 330 Narendra Kumar N V, Shyamasundar R K (Tata Institute of Fundamental Research)

- Mathematical Modelling of Cryptosystems Based on Diophantine Problem with Gamma Superposition Method

Valeriy Osipyan (Kuban State University) 


\section{Keynote Speakers}

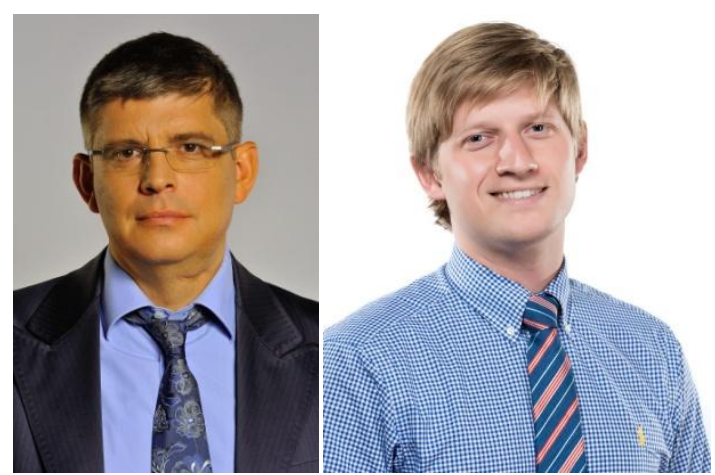

Alexey Markov, Alexander Barabanov NPO Echelon

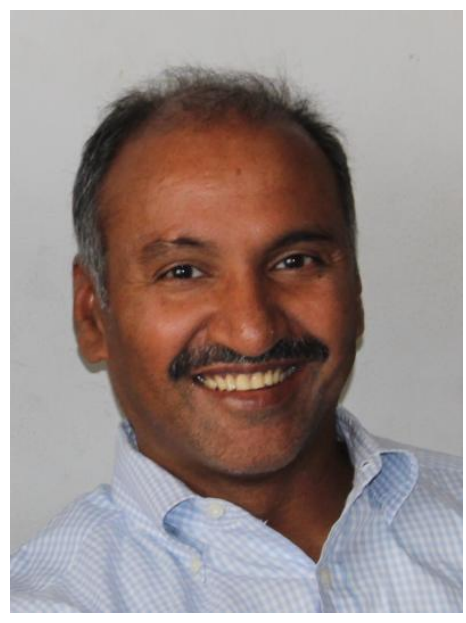

Ramakrishna Thurimella University of Denver

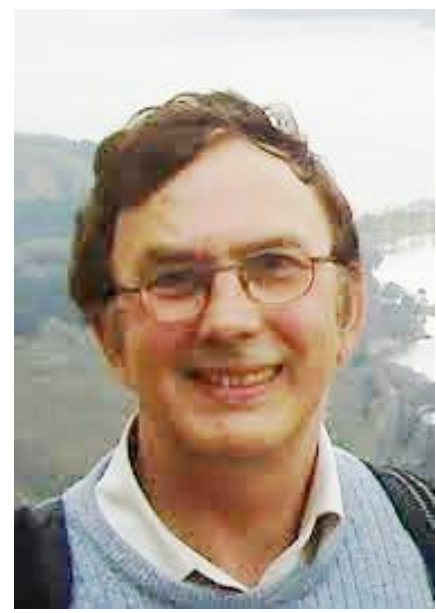

Ron Poet University of Glasgow, UK

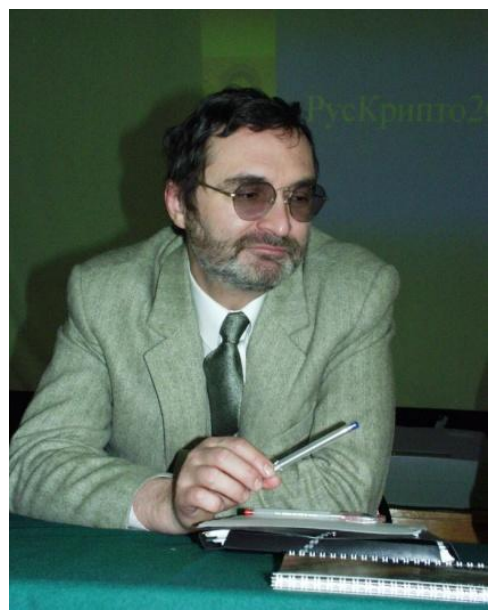

Alexey Zhukov

Bauman Moscow State Technological University 


\section{SIN 2015 Conference Organization}

\section{Honorary Chair: Conference Chairs:}

Program Chairs:

Advisory Committee:

Tutorial Chairs:

Workshop Chairs:

Publication Chairs:

Treasurer:

Registration Chair: Local Arrangements

Chair:
e-Presence Chairs:

Promotion Chairs:

\section{Logo Design:}

Volunteers Team:
Marina Borovskaya, Rector of Southern Federal University, Russia

Oleg Makarevich, Southern Federal University, Russia

Ron Poet, University of Glasgow, UK

Atilla Elçi, Aksaray University, Turkey

Manoj Singh Gaur, Malaviya National Institute of Technology, India

Mehmet Orgun, Macquarie University, Australia

Ludmila Babenko, Southern Federal University, Russia

Sadek Ferdous, University of Glasgow, UK

Anthony T.S. Ho, University of Surrey, UK

Vijay Laxmi, Malaviya National Institute of Technology, India

Josef Pieprzyk, Queensland University of Technology, Australia

Bart Preneel, Katholieke Universiteit Leuven, Belgium

Bülent Örencik, Beykent University, Turkey

Çetin Kaya Koç, University of California Santa Barbara, USA

Edward Dawson, Queensland University of Technology, Australia

Elisa Bertino, Purdue University, USA

N. Balakrishnan, IISc Bangalore, India

Willy Susilo, University of Wollongong, Australia

Bhavani Thuraisingham, University of Texas at Dallas, USA

Evgeny Abramov, Southern Federal University, Russia

Hani Aljahdali, University of Glasgow, UK

Behnam Rahnama, Okan University, Turkey

Dhiren Patel, SVNIT Surat, India

Peter Zegzhda, Saint-Petersburg State Polytechnical University, Russia

Maria Evangelopoulou, University of Glasgow, UK

Hossain Shahriar, Kennesaw State University, USA

Chhagan Lal, SKIT, India

Alexander Chefranov, Eastern Mediterranean University, North Cyprus

Dmitry Rublev, Southern Federal University, Russia

Jaideep Vaidya, Rutgers University, USA

Alexey Nekrasov, Southern Federal University, Russia

Olga Peskova, Southern Federal University, Russia

Maxim Anikeev, Southern Federal University, Russia

Ivan Polovko, Southern Federal University, Russia

Muhammet Saygin, Mersin University, Turkey

Maxim Anikeev, Southern Federal University, Russia

Alexey Nekrasov, Southern Federal University, Russia

Soum Chowdhury, University of Glasgow, UK

Duygu Çelik, Istanbul Aydin University, Turkey

Parvez Faruki, BPTI, Bhavnagar, India

Carlos Becker Westphall, Federal University of Santa Catarina, Brazil

Efe Cem Elçi, Turkey

Nikita Sushkin, Maria Sultanova, Rafael Gevorghian, Maria Tsareva, Students of Southern Federal University and Sochi State University 
Program Committee:
Albert LEVİ, Sabancı University, Turkey

Alessandro BARENGHI, Polytechnic University of Milan, Italy

Alexander BOZHENYUK, Southern Federal University, Russia

Alexander TSELYKH, Southern Federal University, Russia

Alexey TSELYKH, Southern Federal University, Russia

Alfonso RODRIGUEZ, Universidad del Bío-Bío, Chile

Alwyn PAIS, NITK Surathkal, India

Ana Isabel FERRERES, Univ. Carlos III de Madrid, Spain

Anton CHUVAKIN, Security Warrior Consulting, USA

Antonina DURFEE, Royal Bank of Scotland Group USA

Ashutosh SAXENA, Infosys Technologies, India

Attila ÖZGIT, METU, TR

Babak SOKOUTI, Tabriz University of Medical Sciences, Tabriz, Iran

Behnam RAHNAMA, Okan University, Turkey

Bernardi PRANGGONO, Glasgow Caledonian University, UK

Bok-Min GOI, Universiti Tunku Abdul Rahman, Malaysia

Bruhadeshwar BEZAWADA, IIIT Hyderabad, India

Bulent Orencik, BEYKENT University, Turkey

Cecilia BROWN, University of Baltimore

Cheng-Hsien YU, China University of Technology, Taiwan

Christophe FELTUS, Luxembourg Institute of Science and

Technology, Luxembourg

Chuanren LIU, Rutgers University, USA

Cihangir TEZCAN, Middle East Technical University, Turkey

Dai WATANABE, Hitachi, Japan

David G. ROSADO, University of Castilla - La Mancha, Spain

Dmitry MOSKVIN, St.Petersburg State Polytechnical University,

Russia

Dmitry RUBLEV, Southern Federal University, Russia

Dmitry ZEGZHDA, St.Petersburg State Polytechnical University, Russia

Dominik OLSZEWSKI, Warsaw University of Technology, Poland

Dushyant SINGH, MNIT Jaipur, India

Duygu ÇELIK, Istanbul Aydin University, Turkey

Edgar WEIPPL, Security Research, Austria

Elena ALIFANOVA, Rostov State Economic University, Russia

Erkay SAVAŞ, Sabanci University, Turkey

Evgenia ISHCHUKOVA, Southern Federal University, Russia

Franck LEPREVOST, Universite du Luxembourg, Luxembourg

Georges ATAYA, Solvay Brussels School of Econ. Mangt, Belgium

Gerardo PELOSI, Politecnico di Milano, Italy

Girish Keshav PALSHIKAR, Tata Consultancy Services, India

Hai JIN, Huazhong University of Science and Technology, China

Hossain SHAHRIAR, Kennesaw State University, USA

Igor JUKOV, National Research Nuclear University MEPhI, Russia

Ion TUTANESCU, University of Pitesti, Romania

Jan JÜRJENS, Fraunhofer Institute for Software and System Engineering, Germany 
Johan GROSZSCHAEDL, University of Bristol, UK

Joon Sang BAEK, Khalifa University of Science, UAE

Jose ONIEVA, Universidad de Malaga, Spain

Josef PIEPRZYK, Macquarie University, Australia

Khaled SALAH, KFUPM, Saudi Arabia

Luca BREVEGLIERI, Polytecnic University of Milan, Italy

Ludmila FEDORCHENKO, St. Petersburg Institute for Informatics and

Automation, Russia

Luigi LOGRIPPO, Université du Quebec en Outaouais, Canada

Lyudmila BABENKO, Southern Federal University Russia

Manoj BOHRA, MNIT Jaipur, India

Manoj GAUR, MNIT Jaipur, India

Marcello CINQUE, Università degli Studi di Napoli Federico II, Italy

Margarita KNYAZEVA, Southern Federal University, Russia

Marina KROTOFIL, Technische Universität Hamburg-Harburg,

Germany

Maxim ANIKEEV, Southern Federal University, Russia

Maxim KALININ, Saint-Peterburg Polytechnical University, Russia

Md FERDOUS, University of Glasgow

Mert ÖZARAR, METU, Turkey

Nagmeh MORADPOOR, Abertay University, UK

Natalia EVSTIFEEVA, National Research Nuclear University MEPhI,

Russia

Natalia MILOSLAVSKAYA, National Research Nuclear University

MEPhI, Russia

Nikolay Andreevich MOLDOVYAN, St. Petersburg Institute for Informatics and Automation

Nuno ANTUNES, University of Coimbra, Portugal

Parvez FARUKI, MNIT Jaipur, India

Qiong HUANG, City Univ. of Hong Kong, Hong Kong SAR

Rajbir KAUR, LNMIIT, Jaipur, India

Sadek Mehmet CAGLAYAN, Bogazici University, Istanbul

Sambit BAKSHI, National Institute of Technology Rourkela, India

Sattar ALMALIKY, University of Babylon, Iraq

Serif BAHTIYA, Provus A MasterCard Company

Shaoquan JIANG, Univ. of Electronic Science and Tech., China

Smita NAVAL, MNIT Jaipur, India

Stelvio CIMATO, Universitá degli Studi di Milano, Italy

Tang QIANG, University of Luxembourg, Luxembourg

Tariq MAHMOOD, PAF - Karachi Institute of Economics \& Technology, Pakistan

Tuğkan TUĞLULAR, Izmir Inst. of Tech., Turkey

Uma SRINIVASAN, Capital Markets Cooperative Research Center, Australia

Valeriy OSIPYAN, Kuban State University, Russia

Vasileios VLACHOS, Technological Educational Institute of Thessaly, Greece

Vijay LAXMI, MNIT Jaipur, India Vinod P., MNIT Jaipur, India

Victor SKORMIN, Binghamton University, USA 
Vinod P., SCMS School of Engineering and Technology, India

Wojciech MAZURCZYK, Warsaw University of Technology, Poland Xavier BELLEKENS, University of Strathclyde, UK

Zhen JIANG, West Chester Univ. of Pennsylvania, USA 


\section{SIN 2015 Organizers}
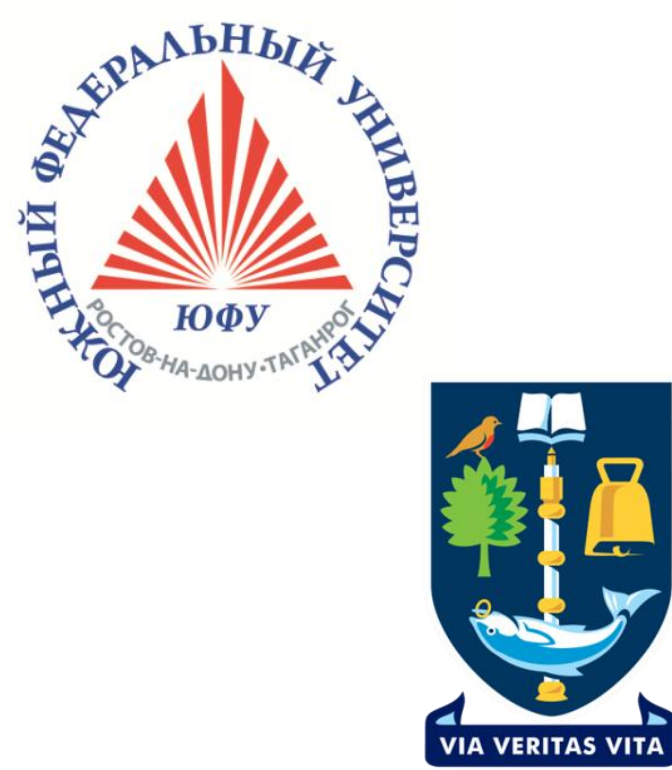
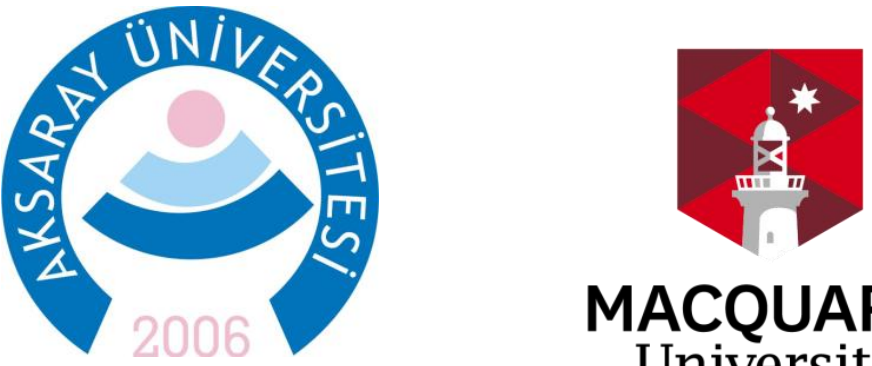

MACQUARIE University

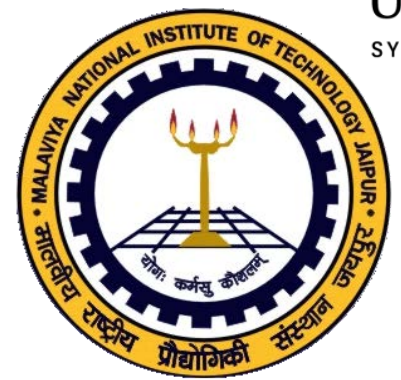

SYDNEY·AUSTRALIA

SIN 2015 Supporter

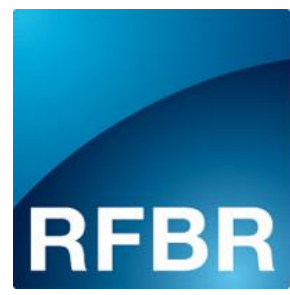

RUSSIAN

FOUNDATION FOR BASIC RESEARCH
SIN 2015 Gold Sponsor

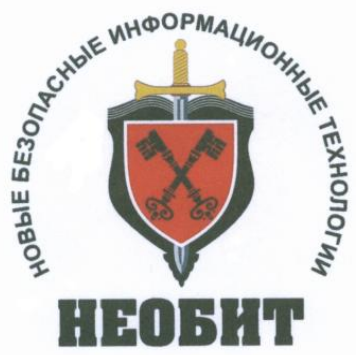

SIN 2015 Silver Sponsor

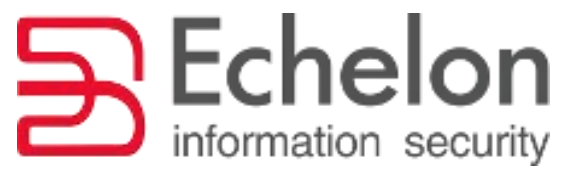

Dedicated to the 100th Anniversary of Southern Federal University

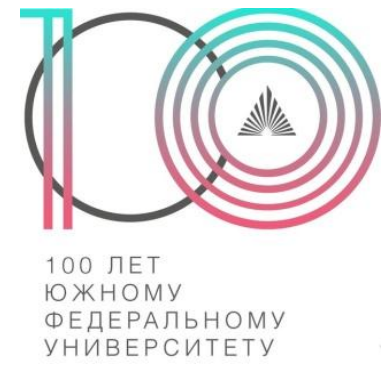

Technical Cooperation 